\title{
Silicone Based Capacitive E-skin Sensor for Soft Surgical Robots
}

\author{
Abu Bakar Dawood ${ }^{1(\bowtie)}$, Hareesh Godaba $^{2}$ and Kaspar Althoefer ${ }^{2}$ \\ ${ }^{1}$ Centre for Advanced Robotics @ Queen Mary, School of Engineering and Materials Science, \\ Queen Mary University of London, E1 4NS, United Kingdom \\ ${ }^{2}$ Centre for Advanced Robotics @ Queen Mary, School of Electronics Engineering and Com- \\ puter Science, Queen Mary University of London, E1 4NS, United Kingdom \\ a. dawoodegmul.ac.uk
}

\begin{abstract}
In this extended abstract, we present a soft stretchable multi-modal capacitive skin sensor that can be used for exteroception and proprioception in soft surgical manipulators. A soft skin prototype was made using Ecoflex, embedding three conductive carbon grease terminal layers. This soft skin is capable of measuring stretch and touch simultaneously. The soft skin measures uniaxial stretches from 1 to 1.2475 within an error range of $2.6 \%$ and can also quantify as well as localize local indentation. An algorithm is developed that decouples local change, i.e., due to indentation, from global strain, due to stretch. An experimental study was conducted; results are presented.
\end{abstract}

Keywords: Capacitive Sensing, Soft Skin, Soft Surgical Robots.

\section{Introduction}

Soft robots, because of their compliance, have a huge potential to be used in Minimally Invasive Surgery (MIS). Their soft structure and variable stiffness allow them to be used in contact with vital organs, without causing any serious damage [1], [2]. However, the compliance of a soft robot, because of its infinite number of degrees of freedom, becomes a challenge when the pose of the robot is to be determined. Apart from proprioception measuring the robot's pose, the interaction of soft robots with the environment or external stimuli is equally important. A soft robot, having a sense of its own position and capability to detect contact with the environment, is important for better control of the robot.

For exteroception, capacitive [3], [4] and resistive [5], [6] methods have been used extensively. Larson et.al [3] studied the effects of stretch and internal pressure on the capacitance of soft capacitive array. However, the capacitance is dependent upon both the pressure and stretch. This coupling between different stimuli has been overlooked.

To detect more than one stimulus simultaneously, different combinations of sensors have been devised, employing different sensing technologies [7]-[11]. However, the idea of multi-modality in soft and stretchable sensors has not been fully explored. 
We present a multi modal soft capacitive skin, that is stretchable and highly compliant and can be integrated into the periphery of a surgical soft manipulator such as the STIFF FLOP manipulator [1], [2] providing feedback on its pose and interaction with the environment for better control during surgery. When the skin is stretched, there is a global change in capacitance, however when a force is applied at a particular point, only a local change would be induced[12]. We propose a decoupling algorithm to distinguish the global sensor stretch from the localised point of force application. Our future work includes quantification of the force applied on a point and application of this skin by wrapping it around a soft robotic actuator.

\section{Materials and Methods}

The material used for the fabrication of our capacitive sensor is Ecoflex 00-30. Moulds for the curing of the Ecoflex sensor layers were 3D printed on an Ultimaker3 3D printer using PLA. Our sensor consists of 4 silicone layers with a thickness of $0.5 \mathrm{~mm}$ each and 3 carbon grease electrode layers, i.e., electrode along X, Y and ground respectively, printed in between these silicone layers.

An experimental setup for stretching was designed using an M8 lead screw driven by a SUNCOR stepper motor. A holder with holes to accommodate for the terminals connecting capacitive skin to the external circuitry, was 3D printed. The capacitance of array sensor was measured using a CAV 424 measurement device by Analog Microelectronics and fed to an Arduino. The $\mathrm{X}$ and Y-terminals were connected to the CAV 424 using two 16-1 Multiplexers, which were switched periodically to record the capacitance of the $10 \mathrm{X}$ and $10 \mathrm{Y}$ terminals.

The unstretched length of our soft skin was $101 \mathrm{~mm}$ and it was stretched from $\lambda=1$ to $\lambda=1.2475$, at increments of $5 \mathrm{~mm}$. A 3D printed spherical indenter of $5 \mathrm{~mm}$ diameter was used for the indentation of the sensor to the following depths: $0 \mathrm{~mm}, 5 \mathrm{~mm}$ and $10 \mathrm{~mm}$, respectively. The experimental setup is shown in Figure 1.
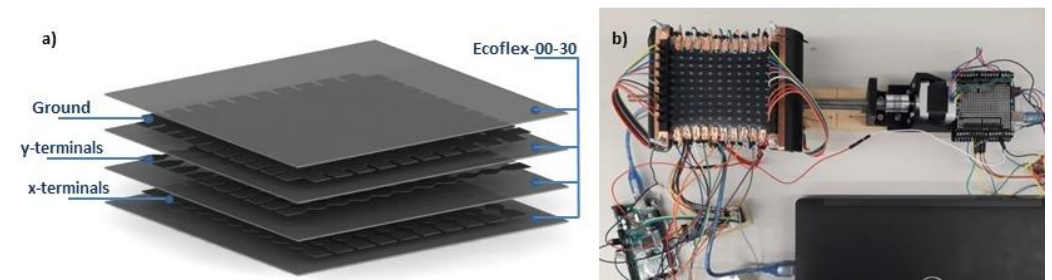

Fig. 1. a) Shows the exploded view of the skin, with $x$, y terminals and ground encapsulated in Ecoflex layers. b) Shows complete experimental setup showing the clamped skin sensor, the mechanism for stretching and the associated sensor signal acquisition circuitry. 


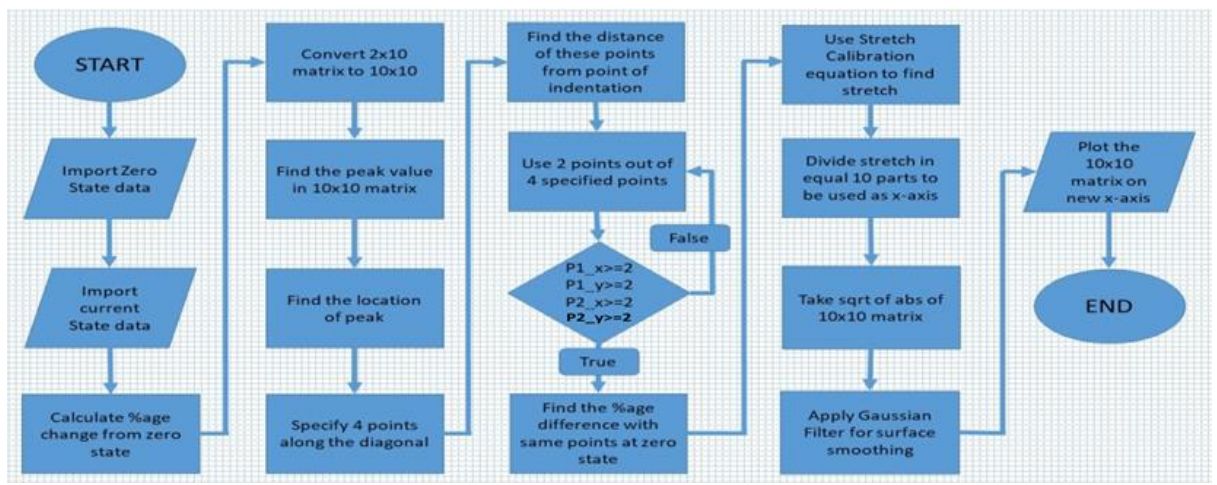

Fig. 2. Flow chart of MATLAB algorithm used for decoupling local indentation and global stretch.

Figure 2 explains the algorithm used for decoupling stretch and indentation. Percentage change in the capacitance values of the 10 horizontal terminals are multiplied by the percentage changes of the 10 vertical terminals to obtain a 10x10 node-matrix. Four heuristic points are defined on the diagonal. The peak value and its coordinates are identified and the distances of peak node from the selected diagonal points are calculated. The two diagonal points having the maximum distance from the peak node are taken into consideration and the corresponding terminals are used to calculate stretch. Average of these two stretches is taken and the data is plotted.

\section{Results}

The sensor skin was stretched while at the same time indentations of different depths were applied. Each state data set was then processed by the algorithm programmed in MATLAB to compute the global stretch. Figure 3 shows two of the datasets processed by using the algorithm. The Y-axis shows the computed stretch while the peak at the point $(6,6)$ in Fig 2 a represents the point of indentation. The point of indentation in Fig $2 \mathrm{~b}$ is $(3,8)$ while the calculated stretch is 1 .
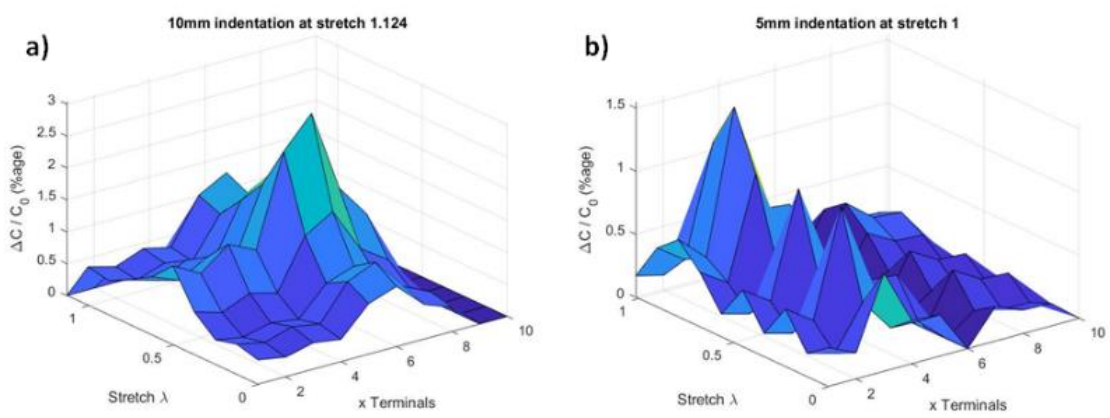

Fig. 3. a) Shows $10 \mathrm{~mm}$ indentation at point $(6,6)$ when the stretch $=1.124$ is applied by the motor. b) Shows $5 \mathrm{~mm}$ indentation at $(3,8)$ when stretch is 1 . 


\section{Conclusion}

The algorithm shows promising results in decoupling global stretch and local indentation. For the investigated cases, a maximum of $2.6 \%$ error was found between the applied stretch and the calculated stretch. Our capacitive skin sensor has shown promising results and future work will include the measurement of the force applied instead of only the location of the indentation. Further, we plan to apply the skin sensor on a soft robot, such as the STIFF FLOP manipulator [1], [2] for sensing the states of deformation and applied external forces for applications in minimal invasive surgery.

\section{References}

[1] J. Fraś, J. Czarnowski, M. Maciaś, and J. Główka, "Static modeling of multisection soft continuum manipulator for stiff-flop project," Adv. Intell. Syst. Comput., 2014, doi: 10.1007/978-3-319-05353-0_35.

[2] M. Cianchetti et al., "Soft Robotics Technologies to Address Shortcomings in Today's Minimally Invasive Surgery: The STIFF-FLOP Approach,” Soft Robot., 2014, doi: 10.1089/soro.2014.0001.

[3] C. Larson et al., "Highly stretchable electroluminescent skin for optical signaling and tactile sensing," Science (80-. )., 2016, doi: 10.1126/science.aac5082.

[4] S. Y. Kim, S. Park, H. W. Park, D. H. Park, Y. Jeong, and D. H. Kim, "Highly Sensitive and Multimodal All-Carbon Skin Sensors Capable of Simultaneously Detecting Tactile and Biological Stimuli," Adv. Mater., 2015, doi: 10.1002/adma.201501408.

[5] J. Park et al., "Giant tunneling piezoresistance of composite elastomers with interlocked microdome arrays for ultrasensitive and multimodal electronic skins," ACS Nano, 2014, doi: $10.1021 / \mathrm{nn} 500441 \mathrm{k}$.

[6] T. Wang et al., "A Self-Healable, Highly Stretchable, and Solution Processable Conductive Polymer Composite for Ultrasensitive Strain and Pressure Sensing," Adv. Funct. Mater., 2018, doi: 10.1002/adfm.201705551.

[7] D. H. Ho, Q. Sun, S. Y. Kim, J. T. Han, D. H. Kim, and J. H. Cho, "Stretchable and Multimodal All Graphene Electronic Skin,” Adv. Mater., 2016, doi: 10.1002/adma.201505739.

[8] Q. Hua et al., "Skin-inspired highly stretchable and conformable matrix networks for multifunctional sensing," Nat. Commun., 2018, doi: 10.1038/s41467-017-02685-9.

[9] P. Maiolino, M. Maggiali, G. Cannata, G. Metta, and L. Natale, "A flexible and robust large scale capacitive tactile system for robots," IEEE Sens. J., 2013, doi: 10.1109/JSEN.2013.2258149.

[10] V. Wall, G. Zoller, and O. Brock, "A method for sensorizing soft actuators and its application to the RBO hand 2," in Proceedings - IEEE International Conference on Robotics and Automation, 2017, doi: 10.1109/ICRA.2017.7989577.

[11] M. Totaro, A. Mondini, A. Bellacicca, P. Milani, and L. Beccai, "Integrated Simultaneous Detection of Tactile and Bending Cues for Soft Robotics," Soft Robot., 2017, doi: 10.1089/soro.2016.0049.

[12] A. B. Dawood, H. Godaba, and K. Althoefer, "Modelling of a soft sensor for exteroception and proprioception in a pneumatically actuated soft robot," in Lecture Notes in Computer Science (including subseries Lecture Notes in Artificial Intelligence and Lecture Notes in Bioinformatics), 2019, vol. 11650 LNAI, pp. 99-110, doi: 10.1007/978-3-030-25332-5_9. 